Research Article

\title{
Skull Sex Estimation Based on Wavelet Transform and Fourier Transform
}

\author{
Wen Yang $\mathbb{( D},{ }^{1}$ Mingquan Zhou $\mathbb{D},{ }^{1,2}$ Pengfei Zhang $\mathbb{D},{ }^{1}$ Guohua Geng $\mathbb{D},{ }^{1}$ Xiaoning Liu $\left(\mathbb{D},{ }^{1}\right.$ \\ and Haibo Zhang $\mathbb{D}^{1}$ \\ ${ }^{1}$ College of Information Science and Technology, Northwest University, Xi'an, China \\ ${ }^{2}$ College of Information Science and Technology, Beijing Normal University, Beijing, China
}

Correspondence should be addressed to Mingquan Zhou; mqzhou@bnu.edu.cn and Xiaoning Liu; 1947776913@qq.com

Received 5 August 2019; Revised 10 December 2019; Accepted 18 December 2019; Published 11 January 2020

Academic Editor: Nikolai E. Lazarov

Copyright (C) 2020 Wen Yang et al. This is an open access article distributed under the Creative Commons Attribution License, which permits unrestricted use, distribution, and reproduction in any medium, provided the original work is properly cited.

\begin{abstract}
Skull sex estimation is one of the hot research topics in forensic anthropology, and has important research value in the fields of criminal investigation, archeology, anthropology, and so on. Sex estimation of skull is crucial in forensic investigations, whether in legal situations that involve living people or to identify mortal remains. The aim of this study is to establish a skull-based sex estimation model in Chinese population, providing a scientific reference for the practical application of forensic medicine and anthropology. We take the superior orbital margin and frontal bone of the skull as the research object and proposed a technology of objective sex estimation of the skull using wavelet transform and Fourier transform. Firstly, the supraorbital margin and frontal bone were quantified by wavelet transform and Fourier transform, and then the extracted features were classified by SVM, and the model was tested. The experimental results show that the accuracy rate of male and female sex discrimination is $90.9 \%$ and $94.4 \%$, respectively, which is higher than that of morphological and measurement methods. Compared with the traditional methods, the method has more theoretical basis and objectivity, and the correct rate is higher.
\end{abstract}

\section{Introduction}

In the practice of forensic identification, it is often the case that a corpse is dismembered and highly decomposed leaving only skeletal remains, or that only skeletal remains are left in a major disaster. It is very important to get the identity information of the dead from the fragmentary bones. Sex estimation is the first and key step of skeletal remains identification, which can reduce the number of possible identity matching by nearly $50 \%$, and also provide basic reference for face reconstruction. Human identity is not only a prerequisite for an individual who officially declares death but also a basis for tracing people, investigating crime, mass disasters, or war atrocities $[1,2]$.

The identification of unknown skeletal remains is an important part of anthropological and forensic research. Sex estimation of unknown bones is a very important part of anthropological and forensic analysis. In the context of forensic analysis, sex estimation together with assessment of racism, population affinity, and stature is important for the process of individual identification. The sex estimation based on skeletal remains is based on sexual dimorphism, which is generally present to varying degrees in bones of human skeleton. Pelvis and skull are the most widely used sites for determining skeletal sex $[3,4]$. However, the pelvis is not easy to store and fragile, and the skull is composed of hard tissue, which in most cases can be well preserved, and the stability of the sexual dimorphic features is better $[5,6]$. Therefore, the skull has become the most commonly used bones in sex estimation, and skull sex estimation has important research significance and application value.

The traditional methods of skull sex estimation are mainly morphological discrimination and measurement discrimination. Morphological discrimination mainly relies on the expert's understanding of the differences in morphological characteristics between male and female skulls. Experts' subjective understanding of skull morphological features has an important influence on sex estimation. The 
measurement discriminant method refers to first identifying the feature points of the skull, measuring some sex-specific feature index items of the skull, and then using these geometric quantities to establish a discriminant function to determine the sex.

In the morphological approach, anthropologists compare visual forms of unknown skulls and draw conclusions through visual observation and experience. Wells [7] used the anthropologists and standard methods in the field of skull sex to assess the sex of the skull. Studies have shown that cognitive bias has an impact on the sex estimation of the skull, and the skull is effective in identifying sex in human skeletal remains. Krogman [8] used morphological methods to identify 750 skulls of known sex. The correct rate was 82-87\%. Ramsthaler et al. [9] used kappa statistics to quantify the differences between two different observers in sex visual morphology assessment, and the consistency was only $90.8 \%$. Therefore, the visual form assessment method is subjective, low in reliability, low in recognition rate, and theoretically insufficient.

The measurement discriminant method is further divided into physical measurement methods and computeraided measurement methods. The physical measurement method is to measure the physical object of the skull and establish a sex discriminant function. Some scholars have done a lot of research using this method and have achieved good results [10-14]. However, the biggest disadvantage of this method is that it may cause secondary damage to the skull during the measurement process, and the measurement results are not accurate enough due to human factors. With the rapid development of computer technology, computer-aided measurement is increasingly used for the measurement of skull feature items. This measurement method is to digitalize the skull object, generate the threedimensional model of the skull, and realize the measurement with the help of computer program or software. At present, the method of computer-aided measurement is the most commonly used method, and many scholars [15-23] use this method to study the sex problem of the skull. Compared with the morphological method, this method is more objective, but the measurement accuracy is still difficult to guarantee. Williams and Rogers [24] showed that when different observers measured some sex differences, most of the measurement variables had errors of more than $10 \%$.

The morphologies of the frontal bone and superior orbital margin are commonly used skull features with sexual dimorphism [23, 25-28]. The male cranial forehead is more inclined, the frontal nodules are not obvious, and the nasal root sag is deeper, while the female cranial forehead is steeper, the frontal node is large and obvious, and the nasal root depression is shallow [23]. Male skulls are characterized by a wider, rounded, "blunt thick" supraorbital margin, while female skulls typically have a thin "sharp, like the edge of a slightly dulled knife" supraorbital margin [29], as shown in Figure 1.
In traditional methods, the accuracy is very low when these two commonly used features are used as skull sex estimation features. Studies have shown that even if two experienced experts use the visual observation method to estimate the sex of the skull, at least $50 \%$ of the observations differ [30]. For these reasons, alternative approaches have been developed based on premises other than simple visual assessment and measurement methods. One of these approaches is the introduction of shape analysis into forensic anthropological, namely, thin-plate splines [31] and Euclidean distance matrix analysis [32]. Alternatively, SchiwyBochat [33] attempted to quantify the roughness of the supranasal (glabellar) region for sex estimation from photographs by applying the box-counting method from fractal geometrics. Although advances have been made in $3 \mathrm{D}$ laser or CT scanning technology, most of the studies use landmark-based or contour-based approaches rather than surface quantification.

Based on the above analysis, we propose a new method for skull sex estimation. In the sex estimation, we first use wavelet analysis to quantify the supraorbital margins of the skull and quantify the frontal sagittal arc using Fourier transformation. Then, we fuse the quantified features and construct a sex classifier using support vector machine method, which ultimately achieves the sex identification of the skull.

Compared with the traditional methods, the innovation and the main contribution of this method are: (1) it gives a clear explanation about the importance of the skull local area for classification, (2) it proposes a promising tool for inexperienced observers to determine the sex of a skull without much human-computer interaction, and (3) it is without tedious manual measurement, quantifies the features, the results are more objective and accurate, and got rid of the influence of the skull size.

The method flow chart of this paper is shown in Figure 2.

\section{Materials}

The skull data used in this study are from Han Adults in northern China. Under the principle of informed consent, the CT images of living human head were obtained by using the multirow detector spiral CT scanner made by Siemens in Germany. The three-dimensional modeling of the skull was realized by computer software developed by ourselves, and the three-dimensional model data of the skull were obtained and saved in $\mathrm{OBJ}$ format. A total of 133 skulls, 73 males and 60 females, aged between 22 and 28, were collected. All skulls are essentially intact; that is, each skull contains all the bones from the parietal bone to the jaw bone and has intact teeth. In addition, their birth date, sex, and residence information were recorded in detail. Patients who had undergone craniofacial surgery, cleft lip or palate or other craniofacial lesions or with syndrome were excluded. This study has been approved by Northwest University ethics committee. Distribution of skulls in male and female of all ages is shown in Table 1. 


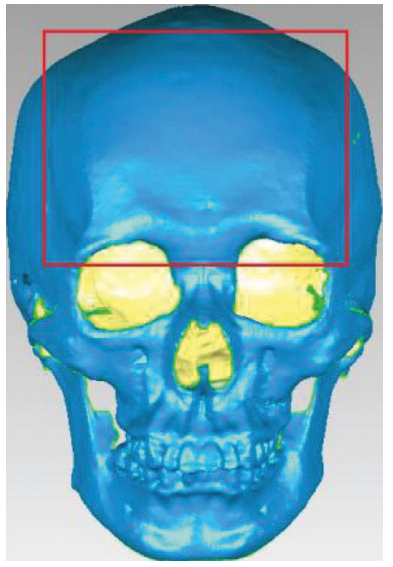

(a)

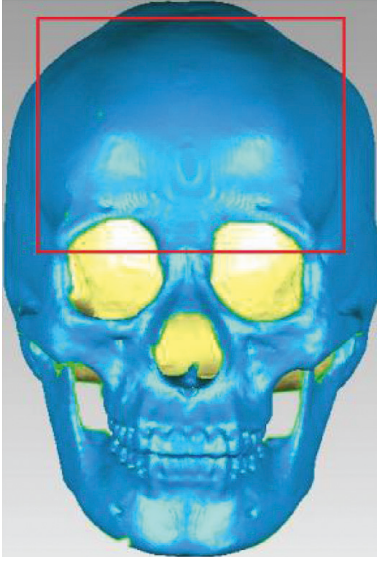

(b)

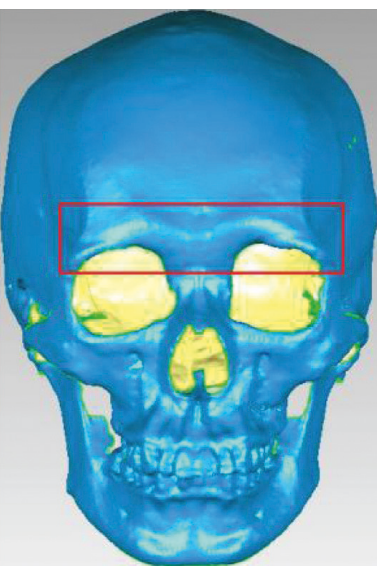

(c)

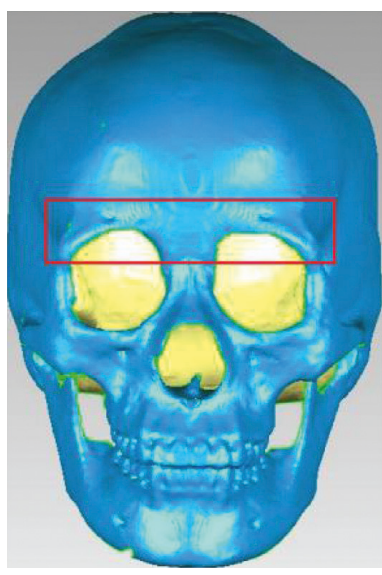

(d)

Figure 1: Difference of frontal bone and supraorbital margin between male and female skulls. (a) Frontal bone of male skull. (b) Frontal bone of female skull. (c) Supraorbital margin of male skull. (d) Supraorbital margin of female skull.

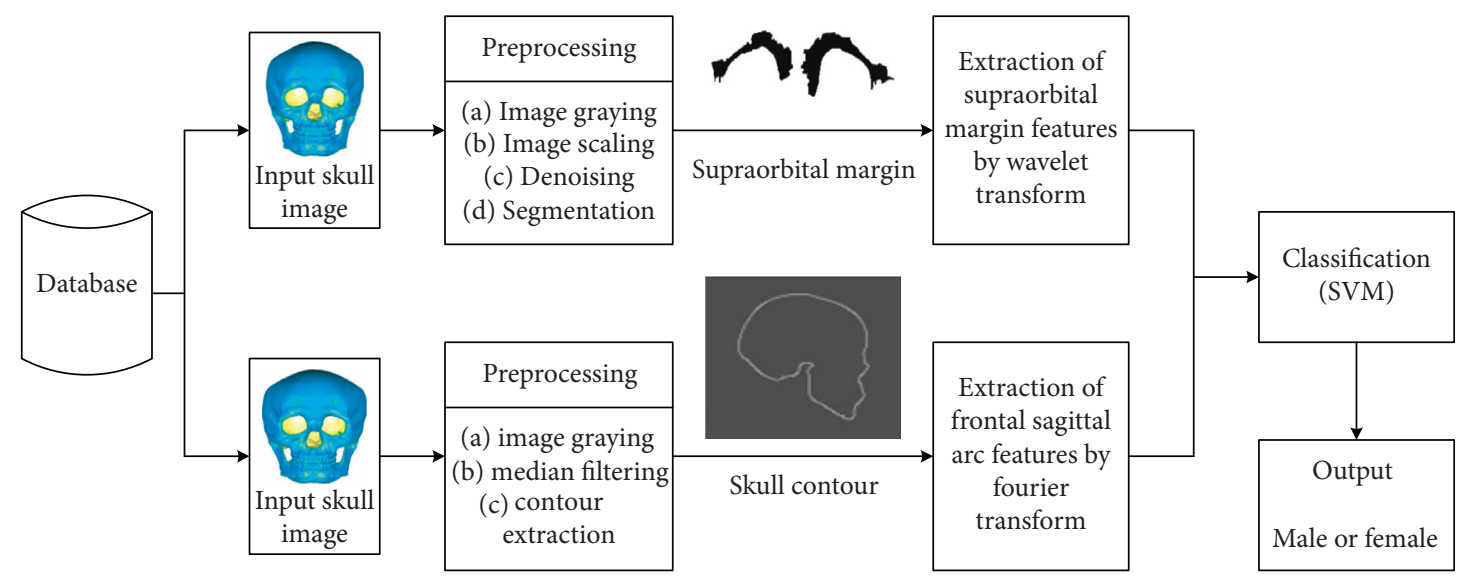

Figure 2: Method flow chart of this paper.

TABLE 1: Distribution of skulls in males and females of all ages.

\begin{tabular}{lccc}
\hline Age (years) & Male & Female & Total \\
\hline 22 & 10 & 7 & 17 \\
23 & 12 & 10 & 22 \\
24 & 14 & 12 & 26 \\
25 & 12 & 10 & 22 \\
26 & 10 & 9 & 19 \\
27 & 8 & 6 & 14 \\
28 & 7 & 6 & 13 \\
Total & 73 & 60 & 133 \\
\hline
\end{tabular}

\section{Methods}

\subsection{Quantitative Extraction of Supraorbital Margin Features}

3.1.1. Segmentation of Supraorbital Margins. The segmentation process of supraorbital margins mainly includes the following steps:

(1) Skull image acquisition: the image used here is a skull image that is completely separated from the skeleton. The skull image data are used in this paper to import the three-dimensional model of the skull into the Geomagic Studio 12.0 software and obtain the frontal image of the skull through the image acquisition function of the software. The supraorbital margins region of all images is complete and clear.

(2) Image gray processing: the skull image obtained in the previous step is a color image, and the color image should be further converted into a gray image. This is because color information has no effect on sex and reduces the amount of calculation.

(3) Image scaling: to ensure accuracy, we scale the image to increase the image pixel rate.

(4) Noise reduction: in the denoising process, the median filter is used to smooth the image, protect the edge information, and increase the image clarity. The main reason to increase the clarity of the image will be clearly able to identify the shape of the supraorbital region for sex estimation of the skull. 
(5) Segmentation: it is the process of extraction of required part of the image. Here, it is necessary to clearly extract the supraorbital margin region to determine the sex of the skull. In this paper, the fuzzy $\mathrm{C}$-means clustering algorithm is used to realize the segmentation process.

3.1.2. Quantitative Extraction of Supraorbital Margins. Let $\mathbf{x}(x, y) \in \mathbf{R}^{2}$ be the vectorial notation of the $2 \mathrm{D}$ point. The continuous wavelet transform (CWT) of a $2 \mathrm{D}$ signal $f(x)$ is defined as follows [34]:

$$
W_{\psi}(\mathbf{b}, a)=\frac{1}{\sqrt{a}} \int \psi^{*}\left(\frac{x-b}{a}\right) f(x) \mathrm{d} x,
$$

where $\psi^{*}, \mathbf{b}$, and $a$ represent the complex conjugate of the analyzed wavelet $\psi$ (or "mother wavelet"), the shifting parameter, and the dilation parameter (related to analyzed scale), respectively.

Depending on the type of information extracted from the signal, choose to use a different mother wavelet. For example, Morlet wavelets are suitable for local frequency analysis, while Mexican hats are suitable for edge detection in image processing. Differential wavelets, such as the derivatives of the Gaussian, are particularly suitable for analyzing singularities and extracting differential information from the signal. Therefore, in this work, we used the first partial derivatives with respect to $x$ and $y$ of the 2D Gaussian function $\phi(x, y)$ denoted as $\psi_{x}$ and $\psi_{y}$ :

$$
\begin{aligned}
& \psi_{x}(x, y)=\frac{\partial \phi(x, y)}{\partial x} \\
& \psi_{y}(x, y)=\frac{\partial \phi(x, y)}{\partial y}
\end{aligned}
$$

where the $2 \mathrm{D}$ Gaussian function is

$$
\phi(x, y)=\exp \left[\frac{-\left(x^{2}+y^{2}\right)}{2}\right]=\exp \left[\frac{-\left(|\mathbf{x}|^{2}\right)}{2}\right]
$$

In order to study morphological variation of supraorbital margin, we have assessed the potential of the wavelet for numerical multiscale estimation of the gradient field of morphological shapes. Therefore, we have applied the 2D wavelet transform $\left(W_{\psi_{x}}\right.$ and $\left.W_{\psi_{y}}\right)$ in the expanded surface for the purpose of calculating the gradient $\nabla W$, i.e.,

$$
\nabla W=\left(W_{\psi_{x}}, W_{\psi_{y}}\right)=\left(\frac{\partial f(x, y)}{\partial x}, \frac{\partial f(x, y)}{\partial y}\right) \text {. }
$$

The gradient-based measurement method has the advantages of translation and rotation invariance, which can ensure that the anatomical correspondence of the supraorbital margin does not depend on the angle and direction of the scanner. The entropy can be measured from the gradient field to achieve a quantitative analysis of the disorder degree of the surface vector direction, i.e., the entropy of the orientation distribution $E_{\nabla W}$, denoted as

$$
E_{\nabla W}=-\sum \rho(\theta) \log \rho(\theta)
$$

where $\rho(\theta)$ is the probability of angle $\theta$ being incremented.

The entropy is maximized when the probability of each measurement is the same; thus, a high entropy value means that the angles of the gradient field are evenly distributed. As discussed earlier, the region of morphologic importance is the valley region of the supraorbital margin, where the high variation of the arrangement of the gradient vectors compared with other regions can be seen in Figure 3.

Considering a small neighborhood of voxels at each point $(x, y)$ on the surface, the entropy is calculated locally, and its value is assigned to the texture value of the supraorbital margin surface. Features are extracted from the surface texture of entropy to describe the degree of sex dimorphism in the supraorbital margin. Further, the valley region of the supraorbital margin is divided by the method based on threshold, which is convenient for subsequent measurements. There are two main thresholds here: one for the surface height value th and another for the entropy te. Two measures were obtained from the segmentation: area and thickness, which was defined as the number of erosions necessary to completely erode the area [35].

\subsection{Quantitative Extraction of Frontal Bone Morphological} Features. After obtaining the skull image using Geomagic Studio 12.0 software, the image is grayscaled and median filtered, and then the processed image is extracted by canny contour to obtain the lateral contour of the skull.

Frontal bones are important regions of skull sex differences. Here, we use Fourier transform to quantify the data of these two nonmeasurable features. Firstly, 18 points were calibrated in the frontal bone regions of skull profile, and the frontal line of three-dimensional skull was fitted by the cftool curve fitting toolbox of MATLAB. Secondly, the spatial curve is optimized by Levenberg-Marquardt algorithm. Finally, the three-dimensional space curve is projected to the two-dimensional plane $X Y$, and the projection curve $S$ is Fourier transformed.

Fourier transform is used to quantify the shape of the curve, i.e., divide the 32 segments on the $X$ axis and then calculate the $Y$ value of the corresponding points on the curve. Apply formula (6) to obtain $A_{0}$, apply formulas (7) and (8) to calculate 16 cosine coefficients $A_{k}$ and 16 sine coefficients $B_{k}$, respectively, and then synthesize into 16 amplitudes $P_{k}$ using formula (9); finally, $P_{k}$ is normalized to $P_{k}^{\prime}$ using formula (10).

$$
\begin{aligned}
& A_{0}=\frac{1}{32} \sum_{m=0}^{31} Y_{m}, \\
& A_{k}=\frac{1}{16} \sum_{m=0}^{31} Y_{m} \times \cos \left(2 \cdot \pi \cdot k \cdot \frac{m}{32}\right), \\
& B_{k}=\frac{1}{16} \sum_{m=0}^{31} Y_{m} \times \sin \left(2 \cdot \pi \cdot k \cdot \frac{m}{32}\right), \\
& P_{k}=\sqrt{A_{k}^{2}+B_{k}^{2}},
\end{aligned}
$$




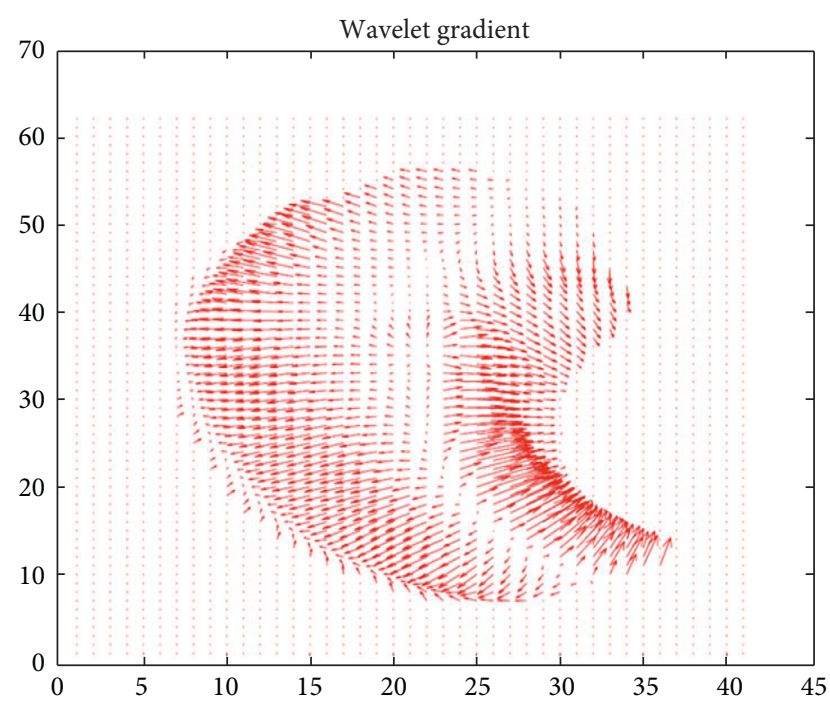

Figure 3: Gradient field of the points (voxels) belonging to the original supraorbital surface.

$$
P_{k}^{\prime}=P \times \frac{100}{A_{0}} \quad(k=1,2, \ldots, 16) .
$$

3.3. Classification Method. Support vector machine (SVM) is a learning algorithm for pattern classification and regression. The basic training principle of SVM is to find the optimal linear hyperplane, which minimizes the expected classification error of unknown test samples, that is, good generalization performance. Because SVM has good learning ability and can solve the problems of small sample, nonlinearity, and high-dimensional classification, it has become the preferred classifier to deal with sex estimation.

3.3.1. Support Vector Classifier. Given a labeled set of $M$ training samples $\left(x_{i}, y_{i}\right)$, where $x_{i} \in R^{N}$ and $y_{i} \in R^{N}$ $y_{i} \in\{-1,1\}$ are associated. SVM classifier finds a small part of the data points of the correct maximum separation hyperplane and maximizes the distance from any class to the hyperplane. Vapnik [36] showed that maximizing the margin is equivalent to minimizing the $\mathrm{VC}$ dimension when constructing the optimal hyperplane. Computing the optimal hyperplane is a constrained optimization problem that can be solved using quadratic programming techniques. The discriminant hyperplane is defined by the level set as follows:

$$
f(x)=\sum_{i=1}^{M} y_{i} \alpha_{i} \cdot k\left(x, x_{i}\right)+b,
$$

where $k(\cdot, \cdot)$ is a kernel function, and the sign of $f(x)$ determines the membership of $x$. Constructing an optimal hyperplane is equivalent to finding all nonzero values $\alpha_{i}$. Any vector $x_{i}$ corresponding to nonzero $\alpha_{i}$ is the support vector of the optimal hyperplane.

For linear SVM, the kernel function is only a simple point product in the input space, while the kernel function in nonlinear SVM effectively projects the sample to a higher (possibly infinite) dimension feature space through the nonlinear mapping function: $\Phi: R^{N} \longrightarrow F^{N}, M \gg N$. Then, construct a hyperplane in $F$. The motive behind this kind of mapping is that it is more likely to find linear hyperplane in high-dimensional feature space. Using Mercer's theorem [37], the samples are projected into the high-dimensional feature space, which can be calculated by the following formula:

$$
k\left(x, x_{i}\right)=\Phi(x) \cdot \Phi\left(x_{i}\right)
$$

where $\Phi(x)$ is the mapping function of projection from lowdimensional space to high-dimensional space and $\cdot$ is the inner product operation.

In order to maximize the separation hyperplane interval $2 /\|w\|$ and minimize the error $\sum_{i=1}^{l} \xi_{i}$ between training samples, the penalty parameter $C$ is introduced [38]. The convex quadratic programming problem can be expressed as follows:

$$
\begin{aligned}
\min _{\gamma, \omega, b} \frac{1}{2}\|\omega\|^{2}+C \sum_{i=1}^{m} \xi_{i}, \xi_{i} \geq 0, \quad i=1, \ldots, m, \\
y^{(i)}\left(\omega^{T} x^{(i)}+b\right) \geq 1-\xi_{i}, \quad i=1, \ldots, m,
\end{aligned}
$$

where $C$ is a constant and $C>0$. When $C$ is larger, it means that the punishment for sex judgment error is larger; when $C$ is smaller, the punishment for sex judgment error is smaller.

In order to obtain the best separating hyperplane in quadratic programming, a Lagrangian operator is constructed to realize the solution, and the following formula is obtained:

$$
\begin{aligned}
\Gamma(\omega, b, \xi, \alpha, r)= & \frac{1}{2} \omega^{T} \omega+C \sum_{i=1}^{m} \xi_{i}-\sum_{i=1}^{m} \alpha\left[y^{(i)}\left(x^{T} \omega+b\right)-1+\xi_{i}\right] \\
& -\sum_{i=1}^{m} r_{i} \xi_{i},
\end{aligned}
$$

where $\alpha_{i}$ and $r_{i}$ are Lagrange multipliers.

Taking formula (14) as a function of variables $\omega$ and $b$, the partial derivatives of them are obtained, and the expressions of $\omega$ and $b$ are obtained. Then, substitute it into formula (14) to find its maximum value. Finally, the following formula is obtained:

$$
\begin{aligned}
\max _{\alpha} W(\alpha)= & \sum_{i=1}^{m} \alpha_{i}-\frac{1}{2} \sum_{j=1}^{m} y^{(i)} y^{(j)} \alpha_{i} \alpha_{j}<x^{(i)}, \\
& x^{(j)}>0 \leq \alpha_{i} \leq C, \quad i=1,2, \ldots, m, \\
\sum_{i=1}^{m} \alpha_{i} y^{(i)} & =0,
\end{aligned}
$$

where $\alpha_{1}, \alpha_{2}, \ldots, \alpha_{m}$ needs to satisfy the conditions of semipositive definite and nonnegative constraints.

\subsubsection{Kernel Function and Optimal Parameter Selection.} The accuracy of sex estimation is directly affected by kernel function. After comparing and analyzing all kinds of kernel 
functions of SVM, radial basis function (RBF) is selected as the kernel function of skull feature mapping. RBF can fit the continuous function on the skull data set as accurately as possible [39], and its mathematical expression can be expressed as follows:

$$
k\left(\left\|x-x_{i}\right\|\right)=\exp \left\{\frac{\left(\left\|x-x_{i}\right\|\right)^{2}}{\delta^{2}}\right\},
$$

where $x_{i}$ is the center of kernel function and $\delta$ is the width of kernel function, which controls the radial action range of kernel function.

In the training stage of sex estimation, parameters $C$ and $\delta$ have the greatest influence on the effect of sex estimation. The change of parameter $C$ can significantly separate the samples with correct classification from those with wrong classification. When $C$ is larger, the classification error rate is smaller, but the interval is smaller; when $C$ is smaller, the interval is larger, but the classification error rate is larger. The change of parameter $\delta$ directly affects the calculation ability of kernel function, thus further affecting the effect of sex estimation. When the $\delta$ is larger, there may be misjudgment, that is, the training samples or test samples are divided into the same category; when the $\delta$ is smaller, it is easy to have over fitting phenomenon, that is, it can correctly classify the sex of the training skull samples, but the classification accuracy of the test skull samples is not high, and the generalization ability is poor. Therefore, it is very important to select the appropriate parameters $C$ and $\delta$ for sex estimation.

The common methods to optimize the parameters $C$ and $\delta$ are grid search, genetic algorithm, and chaos optimization algorithm. In this paper, the algorithm in [40] is used to determine the appropriate parameters $C$ and $\delta$. Set the range of the parameters $C$ and $\delta, 2^{-5} \leq C \leq 2^{15}, 2^{-15} \leq \delta \leq 2^{5}$, and the step size is set to 0.5 to obtain $C$ values and $\delta$ values. The SVM model is used to classify the skull samples and obtain the sex estimation accuracy rate. The optimal parameters $C$ and $\delta$ are determined according to the sex estimation accuracy rate.

Firstly, the quantified supraorbital margin features, frontal bone morphological features, are fused to form the optimal feature set. Then, the SVM classification model is trained according to the optimal feature set and tested. Finally, sex estimation of the unknown skull is realized.

\section{Experiment and Results}

4.1. Fitting Results of Frontal Bone Morphology. We used the method described in Section 3.2 to fit the frontal bone curve, and then projected the frontal bone curve of male and female to the $X Y$ plane. The frontal bone line after projection was obtained as shown in Figure 4.

Using the cftool curve fitting toolbox of MATLAB, the frontal bone lines of male and female were fitted. The fitted curve equations of men and women were as follows:

$y_{1}=-8.6663-1.4380 \cdot x-2.3911 \cdot x^{2}-3.9862 \cdot x^{3}+$ $1.0611 \cdot x^{4}-4.0991 \cdot x^{5}-3.2628 \cdot x^{6}$ and $y_{2}=-16.3129-$ $5.0763 \cdot x-7.2886 \cdot x^{2}+0.1792 \cdot x^{3}-0.0003 \cdot x^{4}+2.0856$.

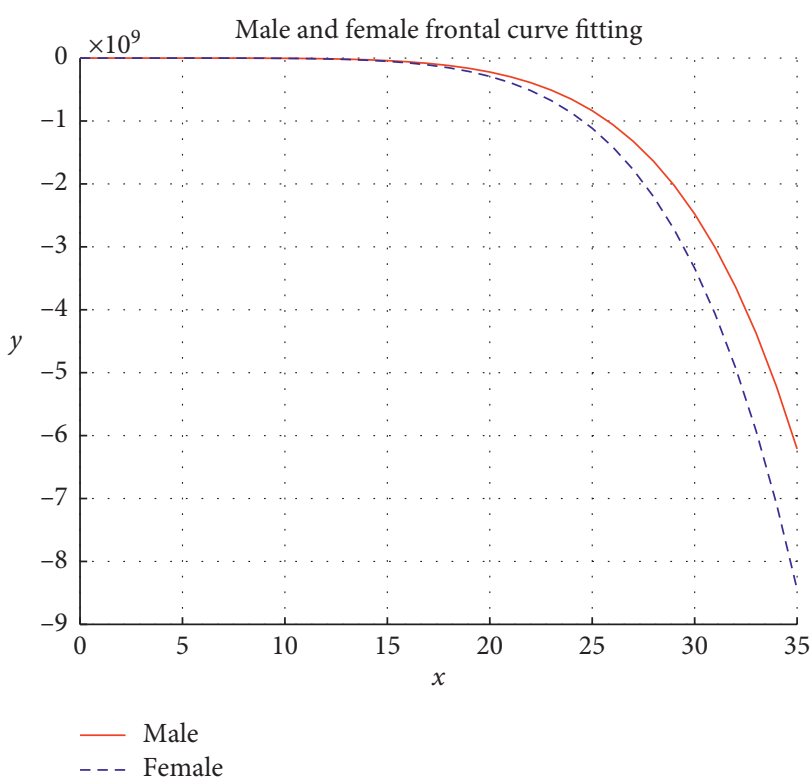

Figure 4: Frontal line diagram.

$x^{5}-4.6519 \cdot x^{6}$ The Fourier transform method is used to quantify the shape of frontal bone lines of male and female. The axes of two-dimensional curves are divided into 32 parts, and the corresponding values on the curves are calculated. Finally, the synthetic amplitude is calculated as the measurement index of sex estimation. Fourier transforms were used for frontal sagittal arc morphology, and a total of 16 sex estimation measurements were obtained.

4.2. Quantitative Results of Supraorbital Margin. We randomly selected 23 skull samples from 133 skulls for testing. Among them, there are 13 males and 10 females. The results are based on measurements of the supraorbital margin valley. The experimental environment is as follows: implemented using Matlab R2015a, all the experimental programs are executed on personal computers with $3.40 \mathrm{GHz}$ CPU and $8 \mathrm{G}$ RAM. Four different values are used for the threshold $t h$, varying from 1 to 4 voxels, which are selected based on observations of the entropy surface. For the threshold te, we used four empirically chosen values: $0.5,0.6,0.7$, and 0.8 . The features were obtained for five different scales of the $2 \mathrm{D}$ wavelet.

Figure 5 presents the two-dimensional feature space defined by the area and thickness of the valley region with respect to the two classes of individuals: male and female. In all parameter combinations, when $t h=2, t e=0.8$, and the scale of the wavelet is 0.00005 , this combination has the highest discriminating power. Under the optimal parameters, these features are most effective in distinguishing between male and female skulls.

4.3. Classification Results. In the sex estimation experiment, we divided the experimental data into two parts, $70 \%$ as training set and $30 \%$ as test set. The SVM method has two important parameters, the penalty parameter $\mathrm{C}$ and the 


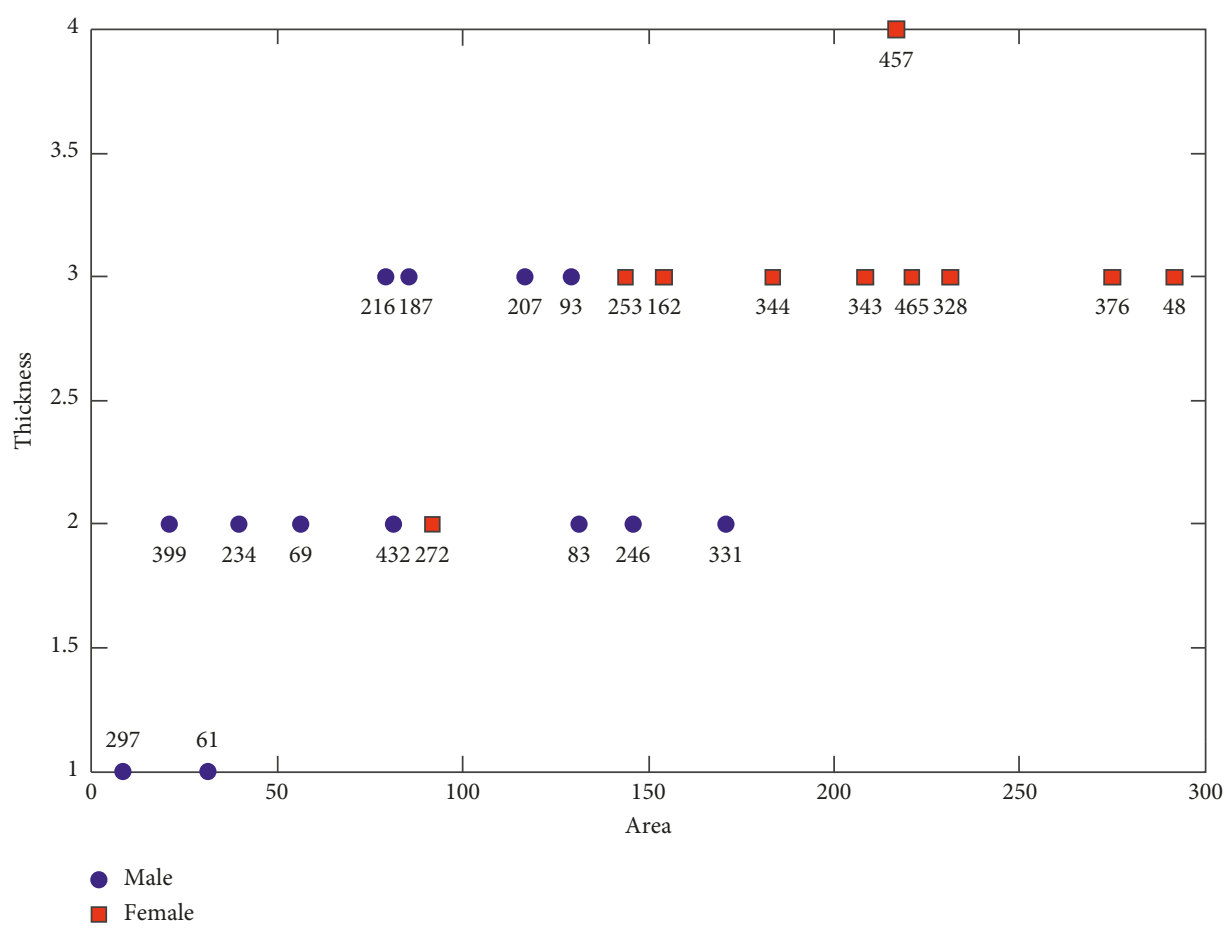

FIGURE 5: Two-dimensional feature space of area and thickness of valley region considering scale is $0.00005, t h=2$ and $t e=0.8$.

parameter A related to the computing power of the kernel function, which have the greatest impact on the results of sex estimation. In this paper, the grid search algorithm is used to solve these two parameters. The range of parameters $C$ and $\delta$ was set to $2^{-5} \leq C \leq 2^{15}, 2^{-15} \leq \delta \leq 2^{5}$, and the step size was set to 0.5 . The optimal parameters $C=1.5824$ and $\delta=0.5$ for 93 training samples (51 males and 40 females) can be obtained by multiple calculations. After the establishment of the sex estimation model, we tested 40 test samples (22 males and 18 females) and compared them with the records in the database. The results were as follows: among 22 male skulls, 2 were misjudged, 20 were correctly judged, and the correct rate was $90.9 \%$; among 18 female skulls, 1 was misjudged, 17 were correctly classified, and the correct rate was $94.4 \%$. The prediction results of the classification model for the test sample are shown in Figure 6.

\section{Discussion}

In forensic medicine and anthropology, researchers use human bones to identify human remains at every stage [41]. The sex of the remains is one of the most important parts of the identification process. Many new methods and technologies have been used in this research, and traditional methods have been constantly improved and optimized. The pelvis and skull are the most commonly used bones in the study of estimating sex by observing and measuring bones [42]. In this study, the measurement of the supraorbital margin of the skull and the morphology of the frontal was used as an indicator of sex. Different from the traditional measurement method, we quantify the indicator by establishing a mathematical model to obtain the measurement

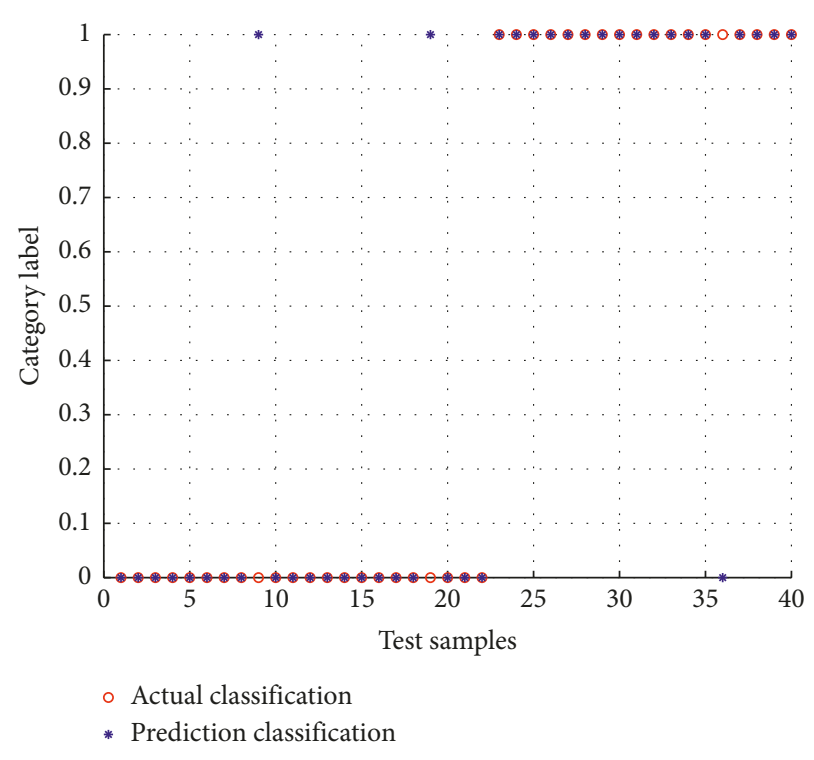

Figure 6: Prediction results of test samples. Label category 0 denotes male and label category 1 denotes female.

result, which overcomes the error of the measurement process.

In the sex estimation problem, when the characteristics of the supraorbital margin and frontal sagittal arcs are used for the study of cranial dimorphism, the measurement results obtained by mathematical modeling are more objective. Since the mathematical model fits the shape, iteratively optimizes and adjusts the parameters to make them closer to the true shape. In the quantification of the sagittal arc feature of the frontal bone, in order to make the Fourier series more 
completely show the shape of the frontal sagittal arc, the subdivision is divided as much as possible, so that the curve formed by the inverse transformation is closer to the original shape. Of course, the finer the division, the more complicated the calculation process. In this study, the frontal sagittal arc was divided into 32 equal parts, and the original curve was reproduced better by inverse transformation, which proved that the degree of division is appropriate. When quantifying the features of the supraorbital margin, the combination of quantifying the supraorbital margin into area and thickness has obvious difference between the two sexes. The results show that the values of female are relatively consistent, and the differences between male are relatively large. This is a common characteristic of sexual dimorphism in the human cranial trait [43]. Bogin's research [44] shows that there is a phenomenon of "prolonged maturation" in the growth of male skeleton. The time and extent of growth are highly variable. Therefore, the range of male supraorbital margins varies widely, and the range of female changes is relatively small.

After quantifying the characteristics of the supraorbital margin and the sagittal arc of the frontal bone, the SVM method was used to establish a sex estimation model, and 40 skulls were tested and achieved good results. In order to verify the superiority of the method, we compare it with the morphological method [8] and the measurement method [21], respectively. The correctness rate of this method is the highest, and the accuracy of the first two methods is less than $90 \%$. The correct rate is over $90 \%$. This is because this study avoids the influence of subjective factors and measurement errors in feature quantification, and the features are more accurate. Therefore, the correct rate is also higher. Liu et al. [45] took the frontal bone as the experimental object and used the forward stepwise regression method based on the maximum likelihood estimation to establish the frontal bone sex discrimination model. The accuracy rate of male discrimination was $89.4 \%$, female discrimination was $85.0 \%$, and the average accuracy rate of discrimination was $87.2 \%$. Yang et al. [46] used Fisher's method and logistic regression method to establish sex discriminant equation for skull with frontal bone only, and the discriminant accuracy was $67.9 \%$ and $68.7 \%$, respectively. $[45,46]$ and this paper use the same anatomical region, but the accuracy of this method is significantly higher than them.

Although the method in this paper has achieved good accuracy in sex estimation, in practical application, the higher the accuracy of sex estimation method, the better the reliability of estimation results. There are still some gaps between the accuracy and ideal value of this paper, which is also the power and direction of future research. In addition, the characteristic areas used for sex estimation in this paper are mainly concentrated in the upper half of the skull. Some researchers have proposed that other areas on the skull can also be used for sex estimation, such as the mandible area $[17,47,48]$ and the occipital area $[16,49]$. Therefore, it is the next research direction to quantify other regions with mathematical methods or machine learning methods and realize skull sex estimation. Although the accuracy rate varies from different researchers, different races, different sample sizes, and different methods, it is of reference value in the application.

\section{Conclusions}

In this study, we took the skulls of Han adults in northern China as the research object, and realized the sex estimation based on skulls. We use the supraorbital margin and frontal sagittal arc of skull as characteristic indicators and use SVM to establish a sex estimation model to achieve sex identification. Firstly, the feature indexes are quantified, the feature of the supraorbital margin is quantified by two-dimensional wavelet transform, and the feature of frontal sagittal arc is quantified by Fourier transform. Then, the quantized features are fused, and the classification model is trained by the SVM method according to the optimal feature set. Finally, the model is tested to verify its performance.

The advantages of this research work are as follows: firstly, it needs no professional qualification; secondly, when quantified, it can fully approximate the true shape of the skull; and finally, it can get a high recognition rate. Although we use CT scanning to construct the 3D point cloud model of skull, this method can also be used to construct the $3 \mathrm{D}$ model in any other way, such as laser scanning and 3D camera. Next, we should collect a larger sample bank to build a model of sex estimation, which will be used in forensic and anthropological fields for the practical application of unknown skeletal sex estimation.

\section{Data Availability}

The .obj format 3D model data used to support the findings of this study may be released upon application to the Northwest University Visual Technology Institute via ghgeng@nwu.edu.cn.

\section{Conflicts of Interest}

The authors declare that they have no conflicts of interest regarding the publication of this paper.

\section{Acknowledgments}

This work was supported by the National Natural Science Foundation of China (nos. 61731015 and 61673319), National Key Research and Development Program of China (no. 2017YFB1402103), Shaanxi Province Industrial Innovation Chain Project of China (no. 2016TZC-G-3-5), Qingdao Municipality's Independent Innovation Major Project of China (no. 2017-4-3-2-xcl), Shaanxi Provincial Natural Science Foundation of China (nos. 2018JM6061 and 2019JQ-166), Shaanxi Provincial Key Research and Development Program General Project (no. 2019SF-272), and Special Program for Natural Science of Shaanxi Provincial Department of Education (nos. 18JK0795 and 19JK0842).

\section{References}

[1] A. Schmeling, G. Geserick, W. Reisinger, and A. Olze, "Age estimation," Forensic Science International, vol. 165, no. 2-3, pp. 178-181, 2007.

[2] A. Schmeling, W. Reisinger, G. Geserick, and A. Olze, "Age estimation of unaccompanied minors-part I: general 
considerations," Forensic Science International, vol. 159, no. 1, pp. S61-S64, 2006.

[3] S. Sangvichien, K. Boonkaew, A. Chuncharunee et al., "Sex determination in Thai skulls by using craniometry: multiple logistic regression analysis," Japanese Journal of Applied Physics, vol. 51, no. 12, pp. 2407-2423, 2007.

[4] A. Bagherpour, M. Imanimoghaddam, M. R. Bagherpour, and M. Einolghozati, "Dental age assessment among Iranian children aged 6-13 years using the Demirjian method," Forensic Science International, vol. 197, pp. 121.e1-121.e4, 2010.

[5] M. Y. Iscan, "Forensic anthropology of sex and body size," Forensic Science International, vol. 147, no. 2, pp. 107-112, 2005.

[6] M. S. Robinson and M. A. Bidmos, "The skull and humerus in the determination of sex: reliability of discriminant function equations," Forensic Science International, vol. 186, pp. 86.e1-86.e5, 2009.

[7] N. Wells, "The impact of cognitive bias in skull sexing," Boston University, Boston, MA, USA, Theses \& Dissertations, 2017.

[8] W. M. Krogman, Book Reviews: the Human Skeleton in Forensic Medicine, Charles C Thomas, vol. 4, pp. 287-288, Springfield, IL, USA, 3rd edition, 1962.

[9] F. Ramsthaler, M. Kettner, A. Gehl, and M. A. Verhoff, "Digital forensic osteology: morphological sexing of skeletal remains using volume-rendered cranial CT scans," Forensic Science International, vol. 195, no. 1-3, pp. 148-152, 2010.

[10] M. R. Dayal, M. A. Spocter, and M. A. Bidmos, "An assessment of sex using the skull of black South Africans by discriminant function analysis," Journal of Comparative Human Biology, vol. 59, no. 1, pp. 209-221, 2008.

[11] A. Amores-Ampuero, "Sexual dimorphism in base of skull," Anthropologischer Anzeiger, vol. 74, no. 1, pp. 9-14, 2017.

[12] Z. Ajanović, A. Sarač-Hadžihalilović, and R. Gojak, "Determination of sex by discriminant function analysis of linear diameters in Bosnian human skulls," in Proceedings of the CMBEBIH 2017: Proceedings of the International Conference on Medical and Biological Engineering, pp. 88-94, Sarajevo, Bosnia, March 2017.

[13] S. Butt and I. Ahmed, "Reliability of cephalogram in determining skull gender dimorphism," Iranian Journal of Orthodontics, vol. 11, no. 1, p. e5321, 2016.

[14] D. A. F. Mary, K. Y. Babu, and K. G. Mohanraj, "Craniometric analysis of foramen magnum with reference to occipital condyles for the determination of sex using dry human skulls," Drug Invention Today, vol. 10, no. 10, pp. 1910-1913, 2018.

[15] D. Franklin, A. Cardini, A. Flavel, and A. Kuliukas, "Estimation of sex form cranial measurements in a Western Australian population," Forensic Science International, vol. 229, no. 1-3, pp. 158.e1-158.e8, 2013.

[16] T. Khaitan, A. Kabiraj, G. Uday, and R. Jain, "Cephalometric analysis for gender determination using maxillary sinus index: a novel dimension in personal identification," International Journal of Density, vol. 2017, Article ID 7026796, 4 pages, 2017.

[17] M. Deng, R. Bai, H. Dong, J. Mu, W. Lin, and Y. Zhou, "Sexual determination of the mandible breadth in a central Chinese population sample: a three-dimensional analysis," Australian Journal of Forensic Sciences, vol. 49, no. 3, pp. 332-343, 2017.

[18] D.-I. Kim, U.-Y. Lee, and S.-H. Han, "Sex determination using three-dimensional image of skull in Korean: metric study by discriminant function analysis," Korean Journal of Physical Anthropology, vol. 28, no. 2, pp. 103-118, 2015.
[19] G. González-Colmenares, C. Sanabria Medina, M. P. RojasSánchez, K. León, and A. Malpud, "Sex estimation from skull base radiographs in a contemporary Colombian population," Journal of Forensic and Legal Medicine, vol. 62, pp. 77-81, 2019.

[20] M. Li, Y. N. Fan, Y. M. Yu et al., "Sex assessment of adult from southwest area of China by bones of facial cranium," Chinese Journal of Forensic Medicine, vol. 27, no. 2, pp. 132-134, 2012.

[21] W. Y. Shui, R. C. Yin, M. Q. Zhou et al., "Sex determination from digital skull model for the Han people in China," Chinese Journal of Forensic Medicine, vol. 28, no. 6, pp. 461-464, 2013.

[22] M. Q. Wang, X. Y. Zhao, P. Bai et al., "Study on sex determination of adult skull in northeast China by CT three-dimensional imaging technique," Chinese Journal of Laboratory Diagnos, vol. 20, no. 4, pp. 671-674, 2016.

[23] Q. N. Zhao, Research on Computer-Assisted Three-Dimensional Skull Sex Identification Method, Northwestern University, Xi'an, China, 2016.

[24] B. A. Williams and T. Rogers, "Evaluating the accuracy and precision of cranial morphological traits for sex determination," Journal of Forensic Sciences, vol. 51, no. 4, pp. 729-735, 2010.

[25] J. A. Keen, "A study of the differences between male and female skulls," American Journal of Physical Anthropology, vol. 8, no. 1, pp. 65-80, 1950.

[26] D. Ferembach, I. Schwindezky, and M. Stoukal, "Recommendations for age and sex diagnoses of skeletons," Journal of Human Evolution, vol. 9, no. 7, pp. 517-549, 1980.

[27] F. W. Rösing, M. Graw, B. Marré et al., "Recommendations for the forensic diagnosis of sex and age from skeletons," HOMO, vol. 58, no. 1, pp. 75-89, 2007.

[28] P. N. Gonzalez, V. Bernal, and S. I. Perez, "Analysis of sexual dimorphism of craniofacial traits using geometric morphometric techniques," International Journal of Osteoarchaeology, vol. 21, no. 1, pp. 82-91, 2011.

[29] R. L. Brooks, "Standards for data collection from human skeletal remains. Arkansas archeological survey research series no. 44 by Jane E. Buikstra; Douglas H. Ubelaker," American Journal of Obstetrics \& Gynecology, vol. 206, no. 1, p. S199, 2012.

[30] P. L. Walker, "Sexing skulls using discriminant function analysis of visually assessed traits," American Journal of Physical Anthropology, vol. 136, no. 1, pp. 39-50, 2008.

[31] E. Pretorius, M. Steyn, and Y. Scholtz, "Investigation into the usability of geometric morphometric analysis in assessment of sexual dimorphism," American Journal of Physical Anthropology, vol. 129, no. 1, pp. 64-70, 2006.

[32] J. T. Richtsmeier, V. Burke Deleon, and S. R. Lele, "The promise of geometric morphometrics," American Journal of Physical Anthropology, vol. 119, no. S35, pp. 63-91, 2002.

[33] K.-H. Schiwy-Bochat, "The roughness of the supranasal region-a morphological sex trait," Forensic Science International, vol. 117, no. 1-2, pp. 7-13, 2001.

[34] F. C. Luciano and M. C. Roberto, Shape Analysis and Classification: Theory and Practice, CRC Press, Boca Raton, FL, USA, 2000.

[35] L. da F. Costa and R. M. Cesar Jr., Shape Classification and Analysis: Theory and Practice, CRC Press, Boca Raton, FL, USA, 2nd edition, 2009.

[36] V. N. Vapnik, The Nature of Statistical Learning Theory, Springer, vol. 8, no. 6, Berlin, Germany, 2000.

[37] R. Courant and D. Hilbert, "Methods of mathematical physics, vol. I," Physics Today, vol. 7, no. 5, p. 17, 1954. 
[38] B. Li, Q. Z. Yao, Z. M. Luo et al., "Gird-pattern method for model selection of support vector machines," Computer Engineering and Applications, vol. 44, no. 15, pp. 136-138, 2008.

[39] Z. J. Tian, Research and Implementation of Cranio-Facial Reconstruction Based on Regional Deformable Model, Northwest University, Xi'an, China, 2011.

[40] X. N. Wang and Z. B. Li, "Determination of kernel function parameters of support vector machine based on grid search," Journal of Ocean University of China (Natural Science Edition), vol. 3, no. 5, pp. 859-862, 2005.

[41] D. C. Dirkmaat, L. L. Cabo, S. D. Ousley et al., "New perspectives in forensic anthropology," American Journal of Physical Anthropology, vol. 47, pp. 33-52, 2007.

[42] W. Yang, X. Liu, K. Wang, J. Hu, G. Geng, and J. Feng, "Sex determination of three-dimensional skull based on improved backpropagation neural network," Computational and Mathematical Methods in Medicine, vol. 2019, 8 pages, 2019.

[43] P. Urbanová, A. H. Ross, M. Jurda, and M.-I. Nogueira, "Testing the reliability of software tools in sex and ancestry estimation in a multiancestral Brazilian sample," Legal Medicine, vol. 16, no. 5, pp. 264-273, 2014.

[44] B. Bogin, "Evolutionary perspective on human growth," Annual Review of Anthropology, vol. 28, no. 1, pp. 109-153, 1999.

[45] X. Liu, X. Liu, L. Zhu, Q. Zhao, and G. Geng, "Sex determination of incomplete skull of han ethnic in China," in Proceedings of the International Conference on Intelligent Computing (ICIG 2017), vol. 10363, pp. 574-585, Springer, Madurai, India, June 2017.

[46] W. Yang, X. Liu, F. Zhu, G. Geng, and K. Li, "Determination of sex discriminant function analysis in Chinese human skulls," Biometric Recognition, vol. 10996, pp. 589-598, 2018.

[47] M. Kumar and S. Lokanadham, "Sex determination \& morphometric parameters of human mandible," International Journal of Research in Medical Sciences, vol. 1, no. 2, pp. 93-96, 2013.

[48] D. Franklin, P. O'Higgins, C. E. Oxnard, and I. Dadour, "Discriminant function sexing of the mandible of Indigenous South Africans," Forensic Science International, vol. 179, pp. 84.e1-84.e5, 2008.

[49] P. K. Singh, D. Tamrakar, S. Karki et al., "Determinaton of sex from the foramen magnum using 3DCT a nepalese study," Kathmandu University Medical Journal, vol. 15, no. 57, pp. 61-65, 2017. 


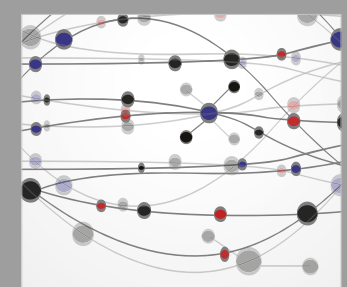

The Scientific World Journal
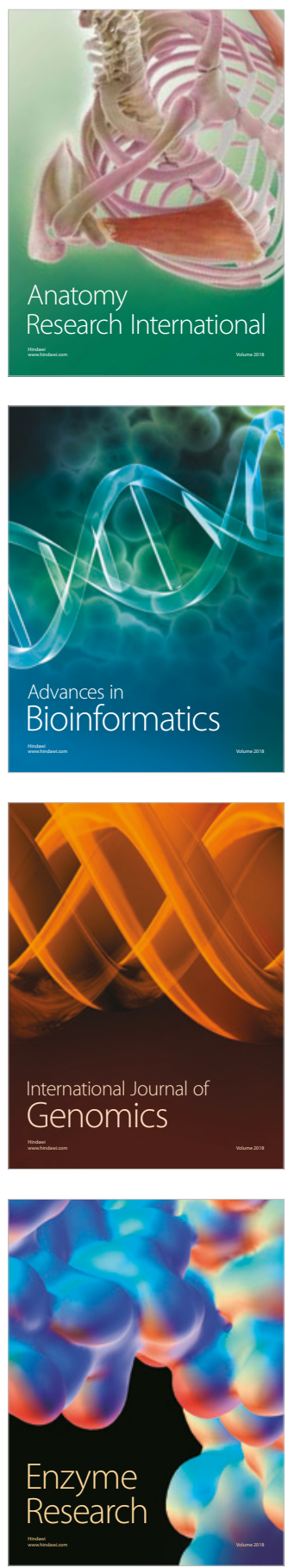
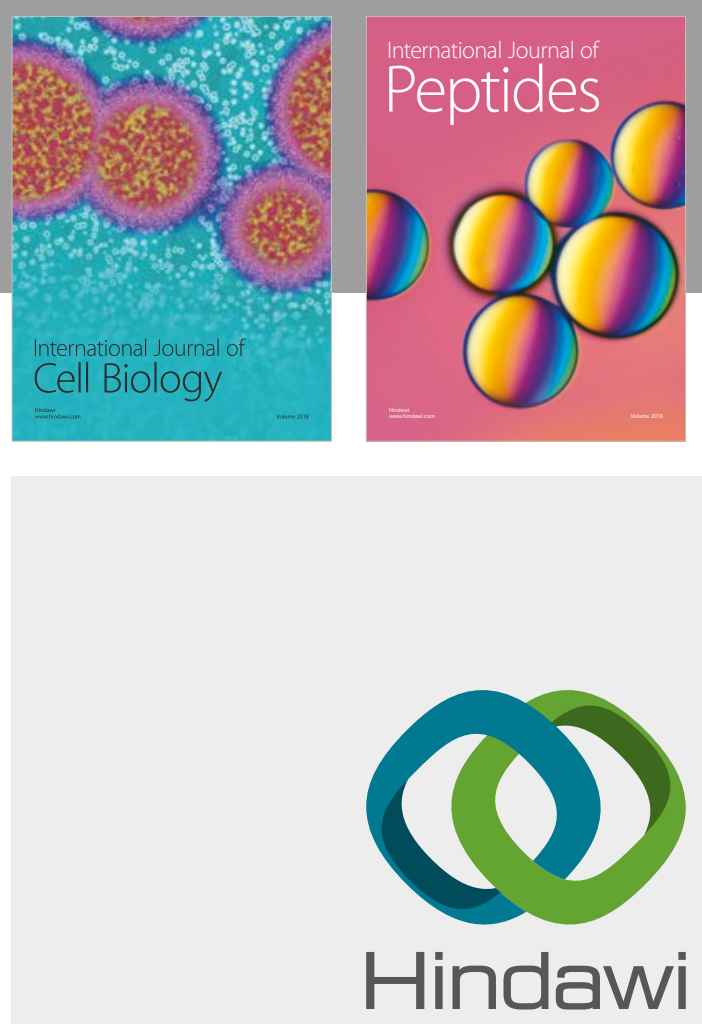

Submit your manuscripts at

www.hindawi.com
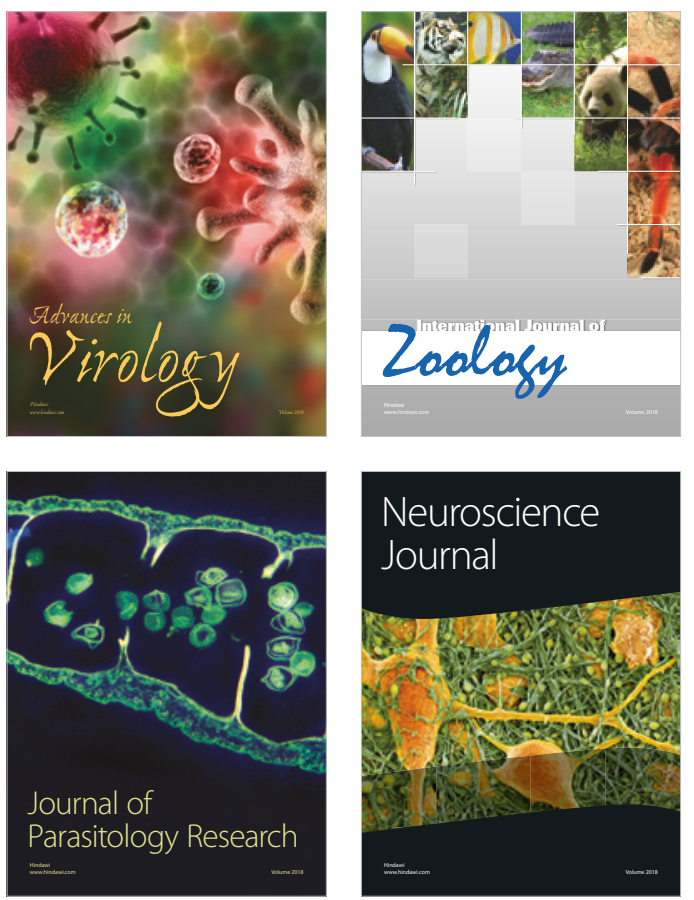
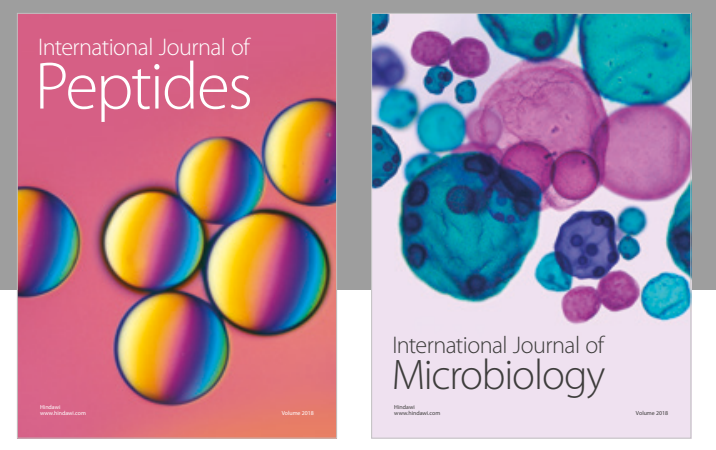

nternational Journal of Microbiology
Journal of
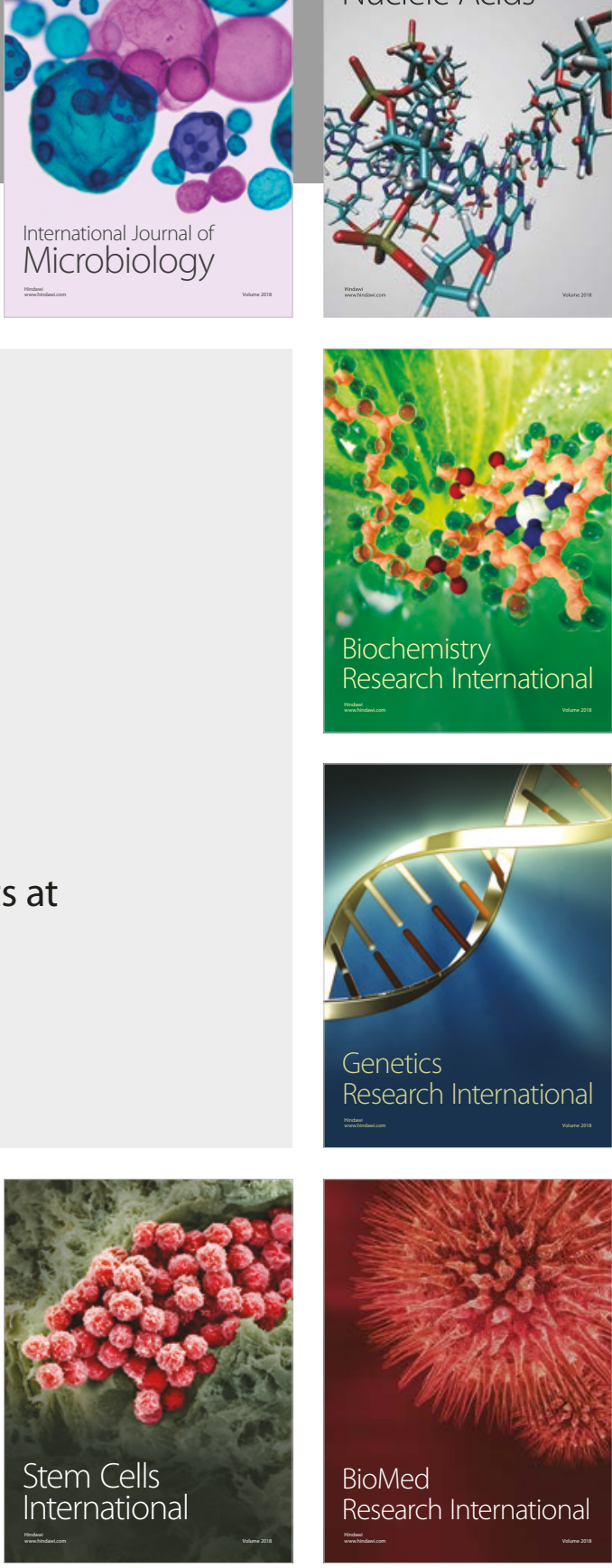
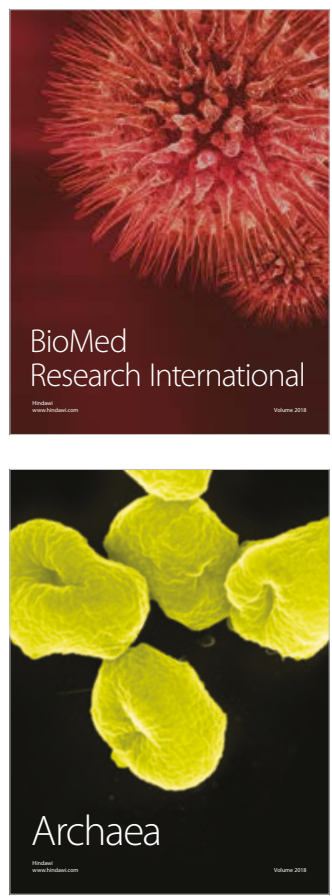